\title{
PRACTICAL SWING-UP CONTROLLER DESIGN FOR A CART-TYPE SINGLE INVERTED PENDULUM HAVING A SERIAL SECOND PENDULUM AS PARASITIC DYNAMICS
}

\author{
Akira Inoue* Mingcong Deng* Masaaki Kosugi* \\ Tomohiro Henmi * \\ * Department of Systems Engineering \\ Okayama University \\ 3-1-1 Tsushima-naka, Okayama 700-8530, Japan \\ \{inoue,deng\}@suri.sys.okayama-u.ac.jp
}

\begin{abstract}
This paper proposes a swing-up control scheme for a cart-type single pendulum having a serial second pendulum as a parasitic part. The proposed control scheme swings up the first pendulum with controlling the motion of a cart and being robust to the parasitic part. In the swing-up, the second pendulum is swinging at the top of the first pendulum and the motion of the second pendulum works as strong disturbance to the control of the first pendulum. Hence the control scheme is required to be strongly robust to the disturbance, and this paper uses sliding mode control law as the controller, which is strongly robust to disturbance. After the swing-up, the controller also stabilizes both angles of the pendulums and cart position. Numerical simulations are given to show the effectiveness of the proposed scheme. Copyright (C)2005 IFAC
\end{abstract}

Keywords: Cart-type pendulum, parasitic dynamics, sliding mode control, swing-up, stabilization

\section{INTRODUCTION}

In general, inverted pendulum system has several types, e.g., a single pendulum (Shiriaev et al., 2001), a double pendulum (Yamakita et al., 1995; Inoue et al., 2004; Henmi et al., 2004), etc.. The use of the inverted pendulum is a traditional topic for verification and practice of various kinds of control theories, because the system is a nonlinear and underactuated mechanical system. Also, it is hard to be controlled by a standard linear

\footnotetext{
1 This research is supported by Grant-in-Aid for Scientific Research from Japan Society for the Promotion of Science (Grant No. 16101005). The authors would like to thank the society for the financial support.
}

controller, so many control methods have been proposed to control the inverted pendulum systems, such as feedback stabilization, energy based control (Wiklund et al., 1993; Åström and Furuta, 2000), bang-bang control, sliding mode control (Inoue et al., 2004), robust control (Yamakita et al., 1995), hybrid control, partial linearization. In spite of these existing methods, to control the inverted pendulum is still an interesting research topic. For example, the serial double inverted pendulum (Henmi et al., 2004; Henmi, 2005) is strongly nonlinear and highly underactuated than a single inverted pendulum and the control of the serial double pendulum is a difficult problem. And a solution of this problem is applicable to 
other nonlinear and underactuated control problems (Fantoni and Lozano, 2002).

For swing-up control of the serial double inverted pendulum, various methods are proposed, e.g., the method in (Yamakita et al., 1995) is a combination of a feedforward controller that swings up pendulums and a feedback controller that stabilizes the pendulum at the upright position. In (Yamakita et al., 1995), a control scheme which transfers the state of pendulum from an arbitrary equilibrium point to another arbitrary equilibrium point is proposed. However, those methods were the controllers for a rotation type pendulum (Kondo and Sugie, 1993; Bortoff, 1996) and they do not consider the restriction to the movement of a carriage. Besides these methods, the controller proposed in (Henmi et al., 2004) swings up and stabilizes the cart-type serial double inverted pendulum and includes control scheme to restrict traveling position of the cart.

However, they do not control the second pendulum while swinging up the first pendulum. This paper considers the serial double pendulum as the single pendulum with parasitic part. In the swingup, the second pendulum is swinging at the top of the first pendulum and the effect of motion of the second pendulum works as strong disturbance to the control of the first pendulumDHence the control scheme is required to be strongly robust to the disturbance, and this paper uses sliding mode control law as the controller, which is strongly robust to disturbance. So far, only in (Inoue et al., 1996), sliding mode controller is used in swingup process. But the controller is not considered the second pendulum.

The proposed controller consists of two steps, Step 1: to swing up the first pendulum, Step 2: to stabilize the first pendulum at the upright position.

In each step, a control scheme and a switching rule from the first step to the next one are given. Although the control law in Step 2 corresponds to the control problem stabilizing the first pendulum in (Inoue et al., 2004; Henmi et al., 2004), the control laws swinging up the pendulum is different from those in (Inoue et al., 2004; Henmi et al., 2004). That is, the proposed controller is given by a swing-up control law in the step 1 with sliding mode control and it has stronger robustness to disturbance.

In Section 2, the dynamics equation is derived for the serial double inverted pendulum system as depicted in Fig.1.

Section 3 is for a controller of Step 1. A control scheme which swings up the first pendulum is given. For swinging up the first pendulum, the sliding mode control is applied.

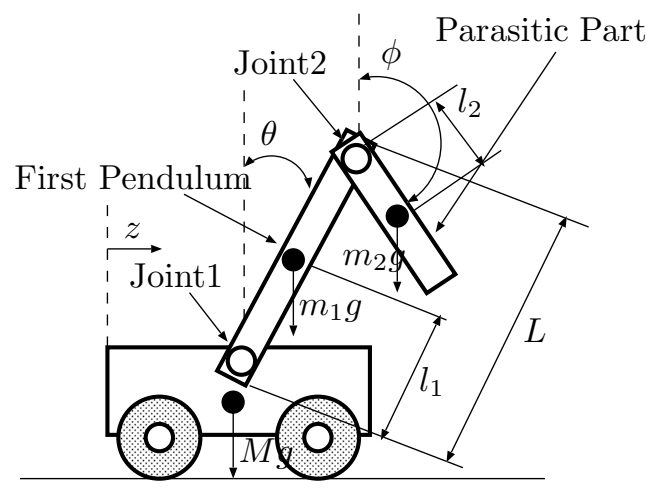

Fig. 1. The serial double inverted pendulum model In Section 4 is for a controller of Step 2. A control law to stabilize the first pendulum with sliding mode control is given.

In Section 5, a numerical simulation is given to show the effectiveness of the proposed scheme in this paper.

\section{DYNAMICS OF THE PENDULUM SYSTEM}

In this paper, the scheme is to swing up a single pendulum. However, the controlled system looks like a serial double pendulum as depicted in Fig. 1. The dynamics of the considered system is modelled by Lagrange method. The parameter of kinetic energy $\left(A_{1}, A_{2}\right)$, potential energy $\left(B_{1}, B_{2}\right)$, and loss energy $\left(C_{1}, C_{2}\right)$ of the first pendulum and the parasitic part, the kinetic energy $\left(A_{3}\right)$ of the cart and the kinetic energy $\left(A_{4}, A_{5}\right)$ of joint of the pendulums are shown as follow.

First Pendulum:

$$
\begin{aligned}
A_{1}= & \frac{1}{2} J_{1} \dot{\theta}^{2}+\frac{1}{2} m_{1}\left\{\frac{d}{d t}\left(z+l_{1} \sin \theta\right)\right\}^{2} \\
& +\frac{1}{2} m_{1}\left\{\frac{d}{d t}\left(l_{1} \cos \theta\right)\right\}^{2} \\
B_{1}= & m_{1} g l_{1} \cos \theta \\
C_{1}= & \frac{1}{2} c_{1} \dot{\theta}^{2}
\end{aligned}
$$

Parasitic Part:

$$
\begin{aligned}
A_{2}= & \frac{1}{2} J_{2} \dot{\phi}^{2}+\frac{1}{2} m_{2}\left\{\frac{d}{d t}\left(z+L \sin \theta+l_{2} \sin \phi\right)\right\}^{2} \\
& +\frac{1}{2} m_{2}\left\{\frac{d}{d t}\left(L \cos \theta+l_{2} \cos \phi\right)\right\}^{2} \\
B_{2}= & m_{2} g\left(L \cos \theta+l_{2} \cos \phi\right) \\
C_{2}= & \frac{1}{2} c_{2}(\dot{\theta}-\dot{\phi})^{2}
\end{aligned}
$$

Cart:

$$
\begin{aligned}
& A_{3}=\frac{1}{2} M \dot{z}^{2} \\
& B_{3}=0
\end{aligned}
$$

Joint between first pendulum and cart:

$$
\begin{aligned}
& A_{4}=\frac{1}{2} I_{n 1} \dot{\theta}^{2}+\frac{1}{2} n_{1} \dot{z}^{2} \\
& B_{4}=0
\end{aligned}
$$


Joint of both pendulums:

$$
\begin{aligned}
A_{5}= & \frac{1}{2} I_{n 2} \dot{\phi}^{2}+\frac{1}{2} n_{1}\left\{\frac{d}{d t}(z+L \sin \theta)\right\}^{2} \\
& +\frac{1}{2} n_{1}\left\{\frac{d}{d t}(L \cos \theta)\right\}^{2} \\
B_{5}= & n_{2} g L \cos \theta
\end{aligned}
$$

Using these equations, the kinetic energy $(A)$, potential energy $(B)$, and loss energy $(C)$ of the serial double inverted pendulum system are given by

$$
A=\sum_{i} A_{i}, \quad B=\sum_{i} B_{i}, \quad C=\sum_{i} C_{i}
$$

Therefore, the Lagrange equation of this system is shown by

$$
\frac{d}{d t} \frac{\partial A}{\partial \dot{x}}-\frac{\partial A}{\partial x}+\frac{\partial B}{\partial x}+\frac{\partial C}{\partial \dot{x}}=0
$$

where $x$ is variable of $(\theta, \phi, z)$.

The dynamics of considered system is given as

$$
\begin{aligned}
& b_{1} \ddot{\theta}+b_{2} \cos (\theta-\phi) \ddot{\phi}+b_{2} \sin (\theta-\phi) \dot{\phi}^{2} \\
& +\left(c_{1}+c_{2}\right) \dot{\theta}-c_{2} \dot{\phi}-b_{3} g \sin \theta+b_{3} \cos \theta \ddot{z}=0(2) \\
& b_{2} \cos (\theta-\phi) \ddot{\theta}+b_{4} \ddot{\phi}-b_{2} \sin (\theta-\phi) \dot{\theta}^{2} \\
& -c_{2} \dot{\theta}+c_{2} \dot{\phi}-b_{5} g \sin \phi+b_{5} \cos \phi \ddot{z}=0(3) \\
& b_{3} \cos \theta \ddot{\theta}+b_{5} \cos \phi \ddot{\phi}-b_{5} \sin \phi \dot{\phi}^{2} \\
& \quad-b_{3} \sin \theta \dot{\theta}^{2}+b_{6} \ddot{z}=f(4)
\end{aligned}
$$

where $b_{1}, b_{2}, b_{3}, b_{4}, b_{5}, b_{6}$ are expressed as

$$
\begin{aligned}
& b_{1}=I_{1}+m_{1} l_{1}^{2}+m_{2} L^{2}+n_{2} L^{2}+I_{n 1} \\
& b_{2}=m_{2} l_{2} L \\
& b_{3}=m_{1} L_{1}+m_{2} L+n_{2} L \\
& b_{4}=I_{2}+m_{2} l_{2}^{2}+I_{n 2} \\
& b_{5}=m_{2} l_{2} \\
& b_{6}=M+m_{1}+m_{2}+n_{1}+n_{2}
\end{aligned}
$$

and the following notations are used through the paper $(\mathrm{i}=1,2)$ :

$z$ : position of the cart

$\theta$ : angular position of the first pendulum from the vertical line

$\phi$ : angular position of the second pendulum from the vertical line

$m_{i}$ : mass of the pendulum or the parasitic part

$n_{i}:$ mass of the joint 1 and 2

$l_{i}$ : length from the $i$ th joint to the center of mass of the pendulum or the parasitic part

$I_{n i}$ : inertia of mass of the $i$ th joint around the center of gravity

$I_{i}$ : inertia of the pendulum or the parasitic part around the joint

$c_{i}$ : viscosity of each joint

$L$ : length of the first pendulum

$g:$ gravity acceleration

$M$ : mass of the cart
The equation for the displacement of the cart $\mathrm{z}[\mathrm{m}]$ is approximated as

$$
f=\ddot{z}
$$

where $f$ is the acceleration command input to the amplifier of the servo motor is the ratio of the attained acceleration $\left[\mathrm{m} / \mathrm{s}^{2}\right]$ to the acceleration command input.

The control objective in this paper is to swing up the first pendulum from the down ward position to the upward position and to stabilize the pendulum at the upward position.

\section{SWING UP THE FIRST PENDULUM WITH CONSIDERING THE PARASITIC PART}

The dynamics of the considering system (2), (3), (4) are arranged as follows.

$$
M(q) \ddot{q}+C(q, \dot{q})+g(q)=F
$$

where

$$
\begin{gathered}
M(q)=\left[\begin{array}{ccc}
b_{1} & b_{2} \cos (\theta-\phi) & b_{3} \cos \theta \\
b_{2} \cos (\theta-\phi) & b_{4} & b_{5} \cos \phi \\
b_{3} \cos \theta & b_{5} \cos \phi & b_{6}
\end{array}\right] \\
C(q, \dot{q})= \\
{\left[\begin{array}{ccc}
c_{1}+c_{2} & -c_{2}+b_{2} \sin (\theta-\phi) & 0 \\
-c_{2}-b_{2} \sin (\theta-\phi) & c_{2} & 0 \\
-b_{3} \sin \theta \dot{\theta} & -b_{5} \sin \phi \dot{\phi} & 0
\end{array}\right]} \\
{\left[\begin{array}{c}
\theta \\
\phi \\
z
\end{array}\right], g(q)=\left[\begin{array}{c}
-b_{3} g \sin \theta \\
-b_{5} g \sin \phi \\
0
\end{array}\right], F=\left[\begin{array}{l}
0 \\
0 \\
f
\end{array}\right]}
\end{gathered}
$$

In the following, the so-called collocated partial feedback linearization method (Spong, 1996) is summarized. By partitioning the vector $q$, we get $q^{T}=\left[q_{1}^{T}, q_{2}^{T}\right]$, with $q_{1}$ corresponding to the passive and $q_{2}$ corresponding to the actuated variables. The Lagrange equations of the dynamics of an $n$-degree of freedom mechanical system with $q_{1}$ passive coordinates and $q_{2}$ actuated coordinates can be redescribed in the following form:

$$
\begin{aligned}
& M_{11} \ddot{q}_{1}+M_{12} \ddot{q}_{2}+C_{1}(q, \dot{q})+g_{1}(q)=0 \\
& M_{21} \ddot{q}_{1}+M_{22} \ddot{q}_{2}+C_{2}(q, \dot{q})+g_{2}(q)=f
\end{aligned}
$$

where

$$
\begin{aligned}
M(q) & =\left[\begin{array}{ll}
M_{11} & M_{12} \\
M_{21} & M_{22}
\end{array}\right], C(q, \dot{q}) \dot{q}=\left[\begin{array}{l}
C_{1}(q, \dot{q}) \\
C_{2}(q, \dot{q})
\end{array}\right] \\
q & =\left[\begin{array}{l}
q_{1} \\
q_{2}
\end{array}\right], g(q)=\left[\begin{array}{l}
g_{1}(q) \\
g_{2}(q)
\end{array}\right]
\end{aligned}
$$

and $M(q)$ is the symmetric, positive definite system inertia matrix. $C$ is the vector of Coriolis and centripetal torques, and $g$ is the vector derived from the potential energy, such as gravitational 
and elastic generalized forces. The vector $f$ represents the input of the generalized forces produced by the $m$ actuators at $q_{2}$ (Yu et al., 1994).

From the property of the inertia matrix, an important property that holds for the entire class of underactuated mechanical systems is the so-called collocated partial feedback linearization property (Spong, 1996). The collocated linearization refers to a control that linearizes the equations associated with the actuated degree of freedom $q_{2}$. Consider the equation (6),

$$
M_{11} \ddot{q}_{1}+M_{12} \ddot{q_{2}}+C_{1}+g_{1}=0
$$

Also, from the uniform positive definiteness of the Matrix $M(q)$, the $l \times l$ matrix $M_{11}$ with $l=n-m$ is invertible. Then, we have

$$
\ddot{q}_{1}=-M_{11}^{-1}\left(M_{12} \ddot{q}_{2}+C_{1}+g_{1}\right)
$$

Further, substituting (10) to equation (8), we obtain

$$
\bar{M}_{22} \ddot{q_{2}}+\bar{C}_{2}+\bar{g}_{2}=f
$$

where

$$
\begin{aligned}
\bar{M}_{22} & =M_{22}-M_{21} M_{11}^{-1} M_{12} \\
\bar{C}_{2} & =C_{2}-M_{21} M_{11}^{-1} C_{1} \\
\bar{g}_{2} & =g_{2}-M_{21} M_{11}^{-1} g_{1}
\end{aligned}
$$

and the $m \times m$ matrix $\bar{M}_{22}$ is symmetric and positive definite. As a result, a partial feedback linearizing controller can be obtained according to equation (11) as follows.

$$
f=\bar{M}_{22} \cdot u+\bar{C}_{2}+\bar{g}_{2}
$$

where $u$ was selected as new control input. The whole system can be rewritten as

$$
\begin{aligned}
M_{11} \ddot{q}_{1}+C_{1}+g_{1} & =-M_{12} u \\
\ddot{q}_{2} & =u
\end{aligned}
$$

Using the collocated linearization method, the original system $(7),(8)$ is feedback equivalent to the system (13), (14).

We consider a swing-up controller for (13) and (14) as follows.

$$
\begin{aligned}
& f=-T_{1} \operatorname{sign}\left(-T_{2} \operatorname{sign}\left(d_{1} \theta+\dot{\theta}\right)\right. \\
& \left.+d_{2} \phi+\dot{\phi}+d_{3} z+\dot{z}\right)(15) \\
& s_{1}=d_{1} \theta+\dot{\theta} \\
& s_{2}=-T_{2} \operatorname{sign}\left(d_{1} \theta+\dot{\theta}\right)+d_{2} \phi+\dot{\phi}+d_{3} z+\dot{z}
\end{aligned}
$$

where, original controller $f$ makes nonlinear system (13) and linear system (14) be stable. Then the values $s_{2}$ and $\dot{s_{2}}$ have different signs. Hence the plane $s_{2}=0$ is reached within a finite time interval. For the motion $d_{2} \phi+\dot{\phi}+d_{3} z+\dot{z}=T_{2} \operatorname{sign}\left(d_{1} \theta+\right.$ $\dot{\theta}$ ), namely $s_{2}=0$, the sliding mode is satisfied by the condition of the linear system $\dot{z}+d_{3} z+$ $d_{2} \phi+\dot{\phi}=\tilde{\tau}$ being controllable by sliding mode control. The main reason is as follows. From the relation between $(\phi, \dot{\phi}, z, \dot{z})$ and $(\theta, \dot{\theta})$ described by system dynamics, instead of $(\phi, \dot{\phi}, z, \dot{z})$, equation $\dot{z}+d_{3} z+d_{2} \phi+\dot{\phi}=\tilde{\tau}$ can be rewritten by $(\theta, \dot{\theta})$ related equation. Then we consider sliding mode controller $\tau=T_{2} \operatorname{sign}\left(d_{1} \theta+\dot{\theta}\right)$ such that the system is stable. The detailed proof of stability is omitted.

According to the above explanation, after a finite time interval the state will reach the intersection of the planes $s_{2}=0$ and $d_{1} \theta+\dot{\theta}=0$. Since $s_{1}=0$, the following first equation is obtained.

$$
\dot{\theta}=-d_{1} \theta
$$

The two-dimensional sliding mode is asymptotically stable, its order is two less than the order of the original system and the motion does not depend on the disturbances (Utkin et al., 1999).

It is worthy to say that when the pendulum swung up in the neighborhood $\theta=0$ by controller (15), the controller switched to the controller of Step 2. That is, $-\theta_{0} \leq \theta \leq \theta_{0}$, where $\theta_{0}$ is a small positive constant. Concerning the reason, besides the reason of stabilizing the first pendulum, the another reason is to avoid the time derivative of $\operatorname{sign}\left(d_{1} \theta+\dot{\theta}\right)$ at $d_{1} \theta+\dot{\theta}=0$.

\section{STABILIZING THE PENDULUM AND CONTROLLING THE CART}

In this section, a control method which stabilizes the pendulum is proposed.

For the stabilizing controller of the pendulum at the unstable equilibrium point, a state feedback stabilization controller is derived by using sliding mode controller (Inoue et al., 2004).

Due to the robustness of the sliding mode controller, the overall system with the parasitic effect can be stabilized by choosing the design parameter by a priori trial and error. In the following, the control law which stabilizes the pendulum is considered. By neglecting $\phi$ in (2), the dynamics of $\theta$ becomes

$$
I_{1} \ddot{\theta}+\left(c_{1}+c_{2}\right) \dot{\theta}-b_{3} g \sin \theta+b_{3} \cos \theta \ddot{z}=0
$$

The control input $f$ is defined as

$$
f=\ddot{z}
$$

The following state variables are chosen as 


$$
\begin{aligned}
x_{2} & =\left[x_{21}, x_{22}, x_{23}, x_{24}\right]^{T} \\
& =[\theta, \dot{\theta}, z, \quad \dot{z}]^{T}
\end{aligned}
$$

With (20) and (21), the linearized state-space equation of (19) around the unstable equilibrium point of the first pendulum $((\theta, \dot{\theta})=(0,0))$ is

$$
\begin{aligned}
\dot{x}_{2} & =A_{2} x_{2}+B_{2} f \\
& =\left[\begin{array}{cccc}
0 & 1 & 0 & 0 \\
\frac{b_{3} g}{b_{1}}-\frac{c_{1}+c_{2}}{b_{1}} & 0 & 0 \\
0 & 0 & 0 & 1 \\
0 & 0 & 0 & 0
\end{array}\right] x_{2}+\left[\begin{array}{c}
0 \\
-\frac{b_{3}}{b_{1}} \\
0 \\
1
\end{array}\right] f
\end{aligned}
$$

A sliding mode controller is used to keep robustness for stabilization of the first pendulum. The control input $f$ which stabilizes (22) is

$$
\begin{aligned}
f & =-\left(S_{2} B_{2}\right)^{-1}\left(S_{2} A_{2} x_{2}+R_{2} \operatorname{sign}\left(\sigma_{2}\right)+K_{2} \sigma_{2}\right) \\
\sigma_{2} & =S_{2} x_{2}
\end{aligned}
$$

where $R_{2}>0, K_{2}>0$ and $S_{2}$ is the solution of the following Riccati equation with $\epsilon_{2}>0$

$$
\begin{aligned}
& P_{2}\left(A_{2}+\epsilon_{2} I\right)+\left(A_{2}+\epsilon_{2} I\right)^{T} P_{2} \\
& \quad-P_{2} B_{2} B_{2}^{T} P_{2}+Q_{2}=0 \\
& S_{2}=B_{2}^{T} P_{2}
\end{aligned}
$$$$
\text { 5. NUMERICAL SIMULATIONS }
$$

In order to show the performance of the proposed scheme in this paper, computer simulation of the swinging up control of the considered system is conducted. The parameters in (2), (3) and (4) are selected to be those of an experimental system of a serial double inverted pendulums in our laboratory. These parameters and the parameters of the control law are given in Table. 1. Simulation result is shown in Fig. 2. Fig. 2 illustrates the responses of $\theta, \dot{\theta}, \phi, \dot{\phi}, z, \dot{z}, u$. The initial states of the system are given by $(\theta(0), \dot{\theta}(0), \phi(0), \dot{\phi}(0), z(0), \dot{z}(0))=$ $(\pi, 0, \pi, 0,0,0)$, and the time when controllers are switched from Step 1 to Step 2 is at the condition of ${ }^{\prime} \cos \theta>0.9^{\prime}$ being satisfied.

Fig. 2 shows that, first, the first pendulum has swung up by (15) of the controller Step 1, and next, the first pendulum has been stabilized at the upright position, the cart has been stabilized at $z=0$ position and parasitic part has been controlled by (23) of the controller of Step 2 .

In Section 4, in order to prove robust stabilization to second pendulum by selecting the design parameter, we undertook another simulation. The result is shown in Fig. 3. It means that a cart's displacement and the parasitic part's oscillation can be controlled by selecting design parameter.

Then, Fig. 4 shows the energy responses when the first simulation is conducted. It illustrates the
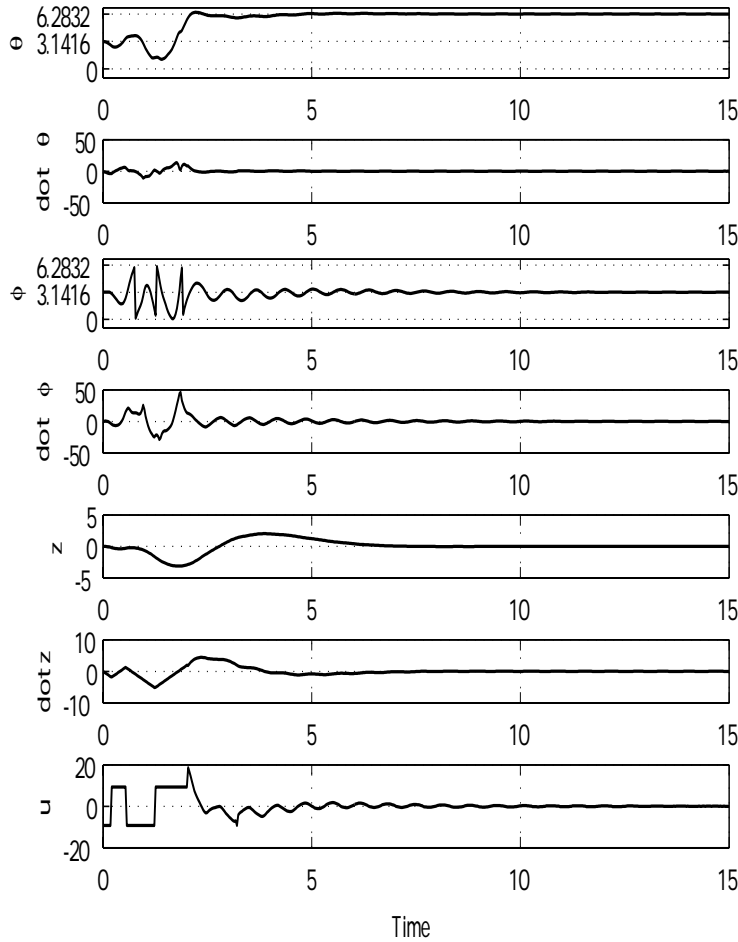

Fig. 2. Time responses of a swing-up control of a serial single pendulum with parasitic $\operatorname{part}\left(K_{2}=1\right)$

energy of the first pendulum $\left(E_{1}\right)$, the parasitic part $\left(E_{2}\right)$ and the cart $\left(E_{3}\right)$.

Table 1. Value of system parameters and control parameters

\begin{tabular}{|l|l||l|l|}
\hline$m_{1}$ & $0.18[\mathrm{~kg}]$ & $m_{2}$ & $0.10[\mathrm{~kg}]$ \\
$n_{1}$ & $0.078[\mathrm{~kg}]$ & $n_{2}$ & $0.05[\mathrm{~kg}]$ \\
$l_{1}$ & $0.105[\mathrm{~m}]$ & $l_{2}$ & $0.115[\mathrm{~m}]$ \\
$J_{n 1}$ & $2.8 \times 10^{-5}\left[\mathrm{kgm}^{2}\right]$ & $J_{n 2}$ & $2.0 \times 10^{-6}\left[\mathrm{kgm}^{2}\right]$ \\
$I_{1}$ & $0.0089\left[\mathrm{kgm}^{2}\right]$ & $I_{2}$ & $0.0018\left[\mathrm{kgm}^{2}\right]$ \\
$c_{1}$ & $0.0001\left[\mathrm{kgm}^{2} / \mathrm{s}\right]$ & $c_{2}$ & $0.002\left[\mathrm{kgm}^{2} / \mathrm{s}\right]$ \\
$L$ & $0.38[\mathrm{~m}]$ & $g$ & $9.8\left[\mathrm{~m} / \mathrm{s}^{2}\right]$ \\
$M$ & $0.44[\mathrm{~kg}]$ & $T_{1}$ & 70.0 \\
$T_{2}$ & 40.0 & $d_{1}$ & 30.0 \\
$d_{2}$ & 30.0 & $d_{3}$ & 70.0 \\
$K_{2}$ & 1.0 & $R_{2}$ & 5.0 \\
\hline
\end{tabular}

\section{CONCLUSIONS}

In this paper, a control scheme which swings up a single inverted pendulum with parasitic part is proposed. This problem is divided into two 


\section{REFERENCES}

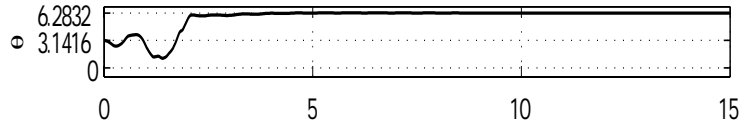

- 50 m ! ! !
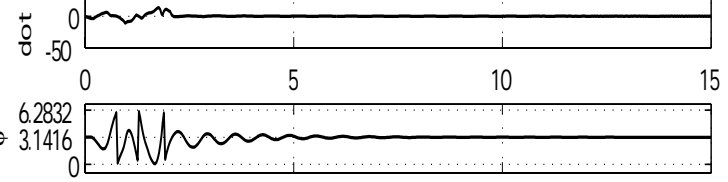

$\begin{array}{llll}0 & 5 & 10 & 15\end{array}$
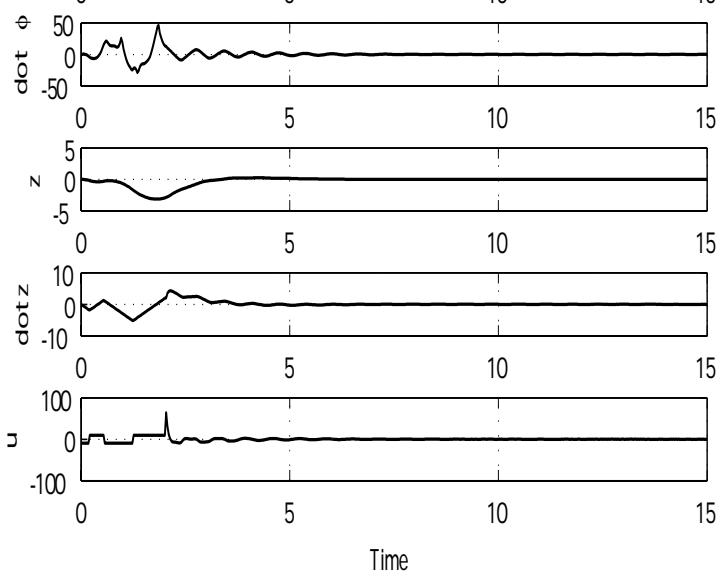

Fig. 3. Time responses of a swing-up control of a serial single pendulum with parasitic $\operatorname{part}\left(K_{2}=20\right)$
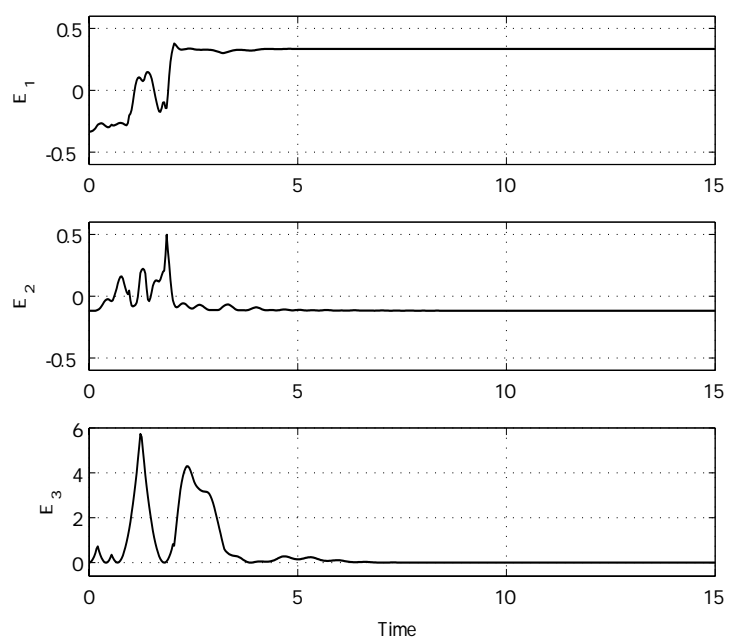

Fig. 4. Energy responses of the first simulation

steps, Step 1 is to swing up the first pendulum considering parasitic part and the position of cart, Step 2 is stabilizing the first pendulum and controlling the cart and parasitic part. Numerical simulations are given to show the effectiveness of the proposed scheme.
Åström, K J. and K. Furuta (2000). Swinging up a pendulum by energy control. Automatica, 36, 287-295.

Bortoff, S A. (1996). Robust swing-up control for a rotational double pendulum. In: Preprints of 13th IFAC world Congress. San Francisco. pp. 413-418.

Fantoni, I. and R. Lozano (2002). Nonlinear Control for Underactuated Mechanical System. Springer.

Henmi, T. (2005). A Research on Nonlinear Control of Underactuated Linked Mechanical Systems. Ph.D Thesis. Okayama University.

Henmi, T., M. Deng, A. Inoue, N. Ueki and Y. Hirashima (2004). Swing-up control of a serial double inverted pendulum. In: Proceedings of the 2004 American Control Conference. Boston. pp. 3992-3997.

Inoue, A., K. Nakayasu and S. Masuda (1996). A swingup control of inverted pendulum using a sliding mode control. In: Proc. of the Third International Conference on Motion and Vibration Control. Chiba. pp. 449-454.

Inoue, A., M. Deng, T. Henmi, N. Ueki and Y. Hirashima (2004). Swingin up contloller design for cart-type double inverted pendulum. Journal of Control Theory and Application, 21, 709-716.

Kondo, T. and T. Sugie (1993). Swing up control of parallel inverted pendulum. In: Preprints of The 20th SICE Symposium on Dynamical System Theory. Sendai. pp. 423-426 (in Japanese).

Shiriaev, A.S., O. Egeland, H. Ludvigsen and A.L. Fradkov (2001). Vss-version of energy-based control for swinging up a pendulum. Systems $\&$ Control Letters, 44, 45-56.

Spong, Mark W. (1996). Energy based control of a class of underactuated mechanical systems. In: Preprints of the 13th IFAC World Congress. Vol. F. pp. 431-435.

Utkin, V., J. Guldner and J. Shi (1999). Sliding Mode Control in Electromechanical Systems. Taylor \& Francis.

Wiklund, M., A. Kristenson and K J. Åström (1993). A new strategy for swinging up an inverted pendulum. In: Preprints of IFAC 12th World Congress. Sydney. pp. 151-154.

Yamakita, M., M. Iwasaki, Y. Sugaharal and K. Furuta (1995). Robust swing up control of double pendulum. In: Proceedings of the American Control Conference. Seattle. pp. 290-295.

Yu, H., L.D. Seneviratne and S.W.E. Earles (1994). Exponentially stable robust control law for robot manipulators. In: IEE Proc.Control Theory. Vol. 141. pp. 389-395. 